\title{
An improved gas distribution system for the ATLAS RPCs
}

\author{
Enrico Pastori ${ }^{12}$ \\ INFN Roma Torvergata \\ Via della Ricerca Scientifica 1, Roma, Italy \\ E-mail: enrico.pastorieroma2.infn.it
}

We present a new gas distribution system for the ATLAS RPCs aimed at equalizing the flow with respect to the average counting rate per gas volume. The system has to take in account the expected luminosity foreseen in superLHC (sLHC) and the resulting wider dynamic gas flow.

XI workshop on Resistive Plate Chambers and Related Detectors (RPC2012) INFN-Laboratori Nazionali di Frascati, Italy

February 5-10, 2012

$\begin{array}{ll}1 & \text { Speaker } \\ 2 & \text { on behalf of the ATLAS MUON collaboration }\end{array}$




\section{Introduction}

The ATLAS RPC gas system consists of 2080 gas units with volumes ranging from 1.36 to 10.771 . The first parameter for the gas-flow equalization is the number of volume exchanges. This parameter has driven the project of the existing gas-distribution system [1] that was planned for increasing the total flow with the rise of the ATLAS background. At the moment, as a consequence of some gas leaks, the gas-flow increment is practically impossible. Almost all of the gas-gap problems noticed until now are related to insufficient gas flow. We also realized that almost all of the found gas leaks are greater than expected due to a misbehavior of the gas distribution at the existing gas-flow rate. For this reason and with a better understanding of the actual ATLAS background we considered the possibility to replace the old gas-distribution system.

\section{Gas flow equalization}

The flow needed in each RPC gas volume is proportional to the prompt charge rate in the gas, which is in turn proportional to the total current measured in each RPC gap (Fig.1). With the data collected in more than one year at a luminosity up to $3 \times 10^{33} \mathrm{~cm}^{-2} \mathrm{~s}^{-1}$ (about $1 / 3$ of the LHC design value), and with a better knowledge of the LHC-induced background [2], it is possible to choose a better gas equalization taking into account the measured charge per gas volume flowing through the detectors.

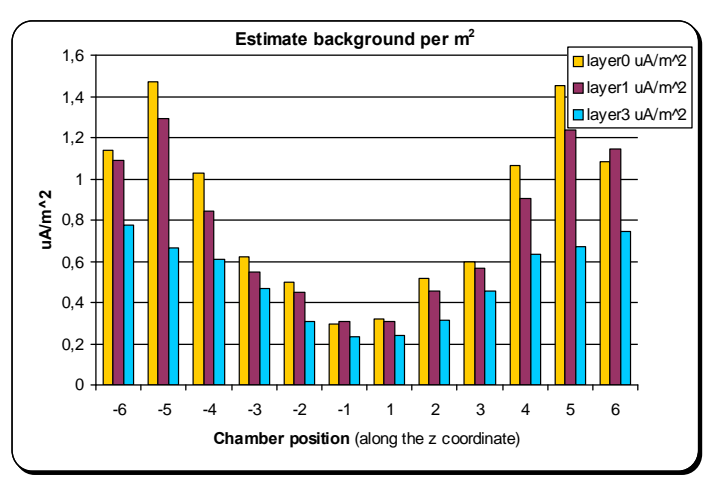

Figure 1: The estimated background was obtained from the current difference measured in each detector with and without the beam interactions, integrated over the all sectors ( $\Phi$ coordinate). Notice the excess on chamber number 5 in both sides.

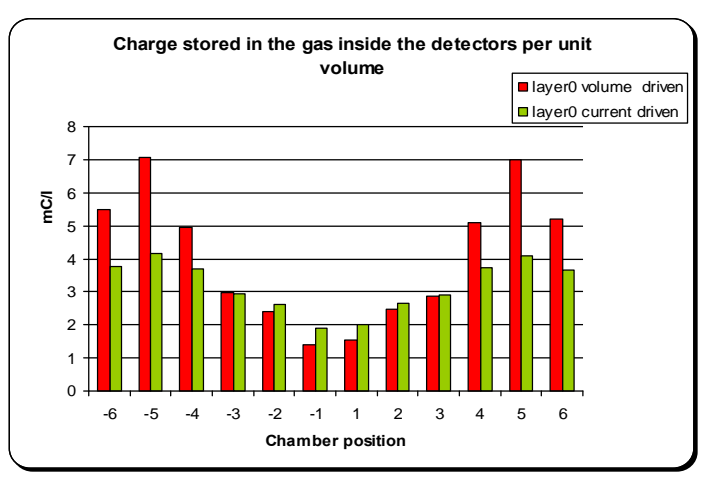

Figure 2: The charge stored in the gas flowing through the detectors decreases significantly with the new current-driven gas distribution (green bar) especially in the peaks (chambers -5 and 5 ).

In any case for safe detector operation a minimum gas flow is required. This minimum was set at 1.5 volume exchanges per hour. 
Taking into account these two constraints, we have re-equalized the gas flow available at the price of a wider dynamic range of fluxes but reducing the average value of the stored charge (Fig.3). This is one of the most important parameters driving the RPCs to malfunctioning and faster ageing.

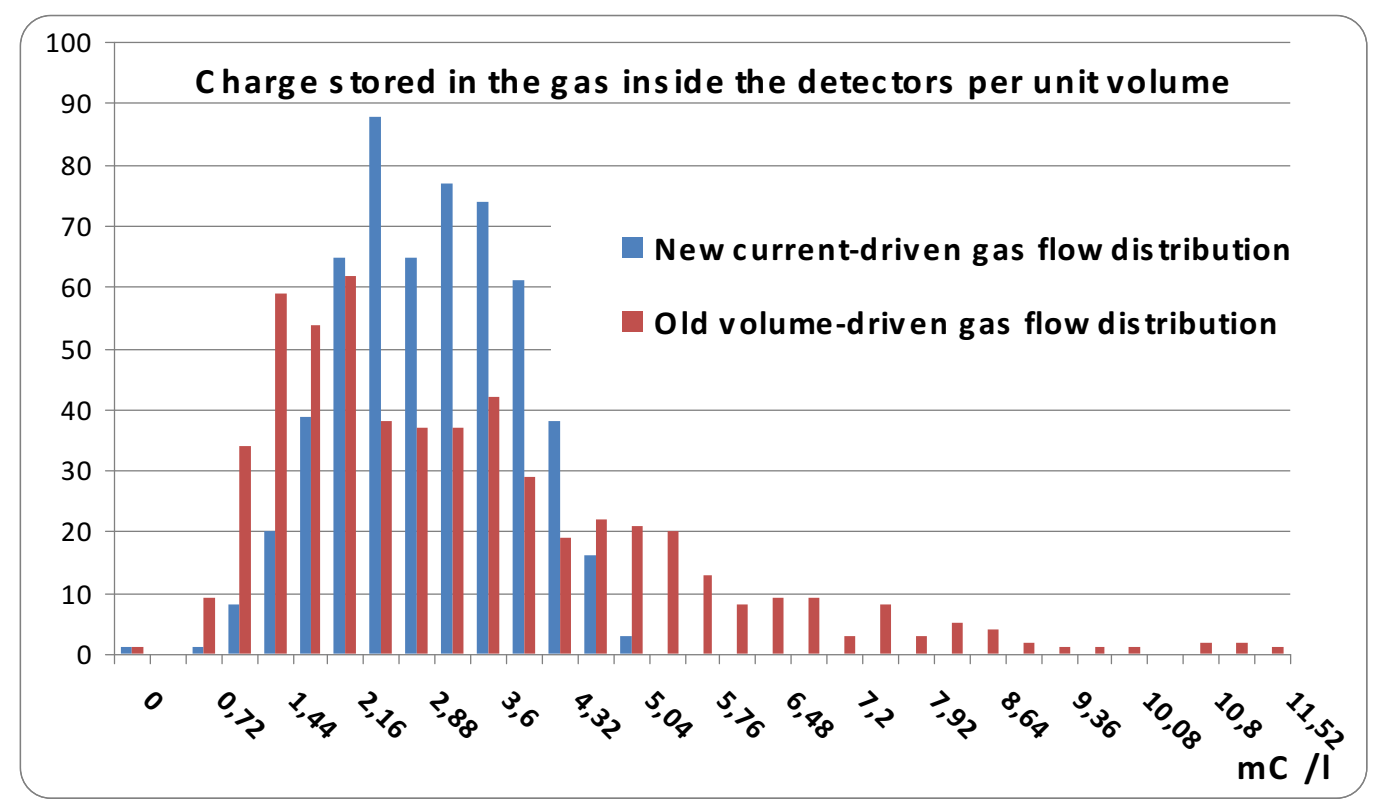

Figure 3: With the new current-driven gas flow distribution the charge stored inside at each detector does not exceed $5.0 \mathrm{mC} / 1$.

\section{Impedances for the gas distribution system}

The starting point in the project of the gas-flow distribution system is the Hagen-Poiseuille law

$$
\Delta P=\frac{8 \mu L}{\pi r^{4}} \Phi
$$

The equation gives the pressure drop $(\Delta P)$ in a fluid with dynamic viscosity $\mu$ flowing through a long cylindrical pipe of length $L$ and radius $r$ with the assumptions that the flow $\Phi$ is laminar, viscous and incompressible (in our case this is true because $\Delta P<<$ atmospheric pressure) and the flow is through a constant circular cross-section that is substantially longer than its diameter. The fluid flow will be turbulent for velocities above a threshold (Reynolds number greater than 2000), leading to larger pressure drops than those expected according to the Hagen-Poiseuille equation.

Hagen-Poiseuille law corresponds to Ohm's law for electrical circuits, where the pressure drop is analogous to the voltage and the volumetric flow rate is analogous to the current because both illustrate transport phenomena. In an electrical circuit starting with a fixed input voltage, it is possible to drive the same current in different loads by connecting a resistance of appropriate value in series, with the assumption that the voltage drop in each resistance will be greater than 
the voltage drop in each load (ideal current generator limit). In the same way, starting from a common pressure rail for a proper flow distribution, a gas impedance delivers a certain flow depending only on its value if the pressure drop in each gas layer will be negligible compared to the pressure drop on the impedance.

We have set the pressure drop in each impedance to 6 mbar which is much greater than the pressure drop in each gas layer plus the return manifold pressure. This value of 6 mbar is well in the range of the setting possibility of the gas racks and is not so high as to reach unsafe conditions in case of blocked outputs in some detectors. Furthermore we are planning to remove the flow-line screw regulation in the gas racks because the smallest pressure drop in each regulator does not permit an adequate adjustment in the flow distribution, a task which is already well carried out by the gas impedances.

The wide gas flow spread needed in the system requires the same spread in the impedance value: 18 types of impedance were built. For the construction of these impedances, a set of commercial dosage needles with a very strict tolerance of the inner diameter was chosen (the gas impedance depends on the fourth power of the inner diameter).

The impedance was built by jamming the needle inside a Polyurethane (PU) plastic gas pipe (inner diameter $2 \mathrm{~mm}$ ). A sample of each type of needles has been tested with an accurate differential electronic manometer and with a gas flow meter after accurate calibration (Fig.4). The test result (Tab. 1) differs from the Hagen-Poiseuille law for the presence of a quadratic term (Fig.5). This term is due to the sharp change in the gas velocity, as it happens in a bent pipe or in an abrupt change of the pipe diameter.

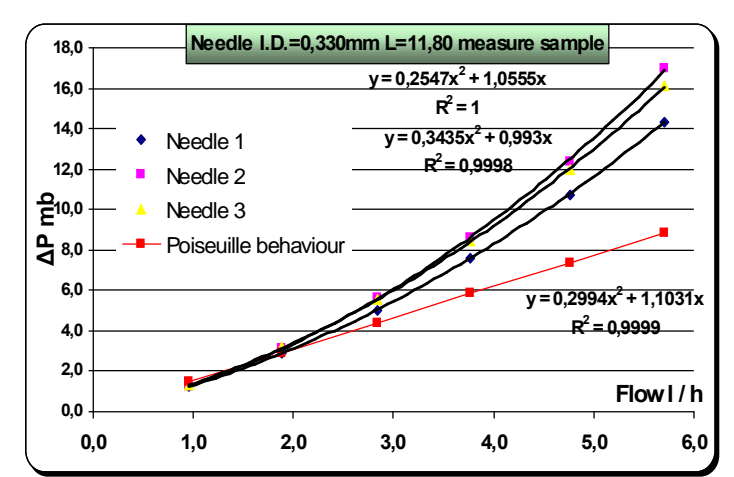

Figure 4: This graph shows the measurements on a sample of needles of the same type. All measurements were done with pure TFE ( $\mu$ very closed to the actual ATLAS gas mixture) and temperature in the range: $19^{\circ} \mathrm{C}-22^{\circ} \mathrm{C}$.

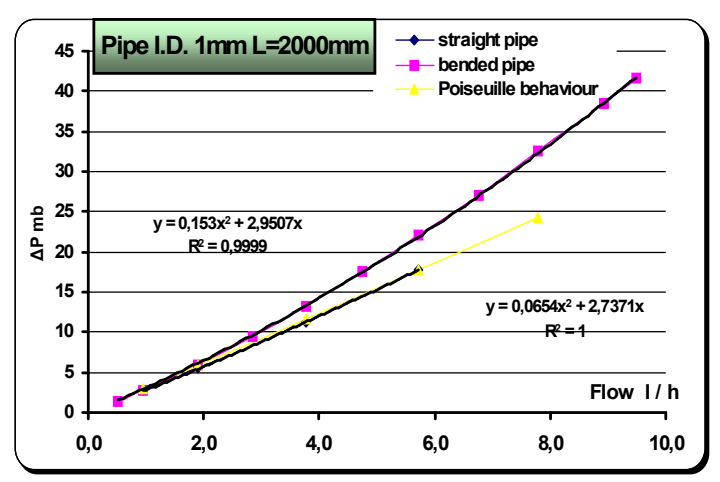

Figure 5: This graph shows the different behaviour for the same capillary straight and bended.

\begin{tabular}{|l|c|c|c|c|c|c|c|c|c|c|c|c|c|c|c|}
\hline Needle Type & $\mathbf{1}$ & $\mathbf{2}$ & $\mathbf{5}$ & $\mathbf{6}$ & $\mathbf{7}$ & $\mathbf{8}$ & $\mathbf{9}$ & $\mathbf{1 0}$ & $\mathbf{1 1}$ & $\mathbf{1 2}$ & $\mathbf{1 3}$ & $\mathbf{1 5}$ & $\mathbf{1 6}$ & $\mathbf{1 7}$ & $\mathbf{1 8}$ \\
\hline DI (mm) & 0,254 & 0,254 & 0,305 & 0,330 & 0,330 & 0,330 & 0,330 & 0,406 & 0,406 & 0,406 & 0,406 & 0,508 & 0,508 & 0,508 & 0,508 \\
Length (mm) & $\mathbf{1 1 , 8 0}$ & $\mathbf{1 8 , 2 0}$ & $\mathbf{1 9 , 2 0}$ & $\mathbf{1 1 , 8 0}$ & $\mathbf{1 8 , 2 0}$ & $\mathbf{3 0 , 8 0}$ & $\mathbf{4 3 , 1 0}$ & $\mathbf{1 1 , 8 0}$ & $\mathbf{1 8 , 2 0}$ & $\mathbf{3 0 , 8 0}$ & $\mathbf{4 3 , 1 0}$ & $\mathbf{1 1 , 8 0}$ & $\mathbf{1 8 , 2 0}$ & $\mathbf{3 0 , 8 0}$ & $\mathbf{4 3 , 1 0}$ \\
Quadratic term & $\mathbf{0 , 8 0 2}$ & $\mathbf{1 , 0 8 3}$ & $\mathbf{0 , 6 7 6}$ & $\mathbf{0 , 2 9 9}$ & $\mathbf{0 , 3 3 2}$ & $\mathbf{0 , 3 4 2}$ & $\mathbf{0 , 4 8 6}$ & $\mathbf{0 , 1 2 9}$ & $\mathbf{0 , 1 2 0}$ & $\mathbf{0 , 1 6 4}$ & $\mathbf{0 , 1 6 9}$ & $\mathbf{0 , 0 6 0}$ & $\mathbf{0 , 0 6 4}$ & $\mathbf{0 , 0 6 4}$ & $\mathbf{0 , 0 8 9}$ \\
Linear term & $\mathbf{2 , 9 2 0}$ & $\mathbf{4 , 0 7 3}$ & $\mathbf{2 , 7 3 1}$ & $\mathbf{1 , 0 5 1}$ & $\mathbf{1 , 8 1 3}$ & $\mathbf{2 , 5 7 0}$ & $\mathbf{4 , 1 2 0}$ & $\mathbf{0 , 6 4 9}$ & $\mathbf{0 , 7 7 5}$ & $\mathbf{1 , 3 2 5}$ & $\mathbf{1 , 8 6 8}$ & $\mathbf{0 , 2 9 1}$ & $\mathbf{0 , 3 6 8}$ & $\mathbf{0 , 6 1 0}$ & $\mathbf{0 , 7 3 5}$ \\
\hline
\end{tabular}

Table 1: This table shows the linear and quadratic terms measured for a sample of each type of needles. 
The flow driven for each needle doubling the starting point pressure from $6 \mathrm{mb}$ to $12 \mathrm{mb}$ is increased only by a factor 1.6 (Tab. 2). This limit is almost insurmountable and is caused by the quadratic term in the $\Delta \mathrm{P}$ vs Flow relation. To overcome this problem we are studying a different type of gas impedance made of many pipes mounted in a parallel configuration. In any case we are confident that with this new gas equalization a good detector operation at the full-project ATLAS background will be possible without increasing the total gas flow more than a factor two.

\begin{tabular}{|c|c|c|c|c|c|c|c|c|c|c|c|c|c|c|c|c|}
\hline Flow(6mb) & & 1,47 & 1,13 & 1,58 & 3,05 & 2,32 & 1,87 & 1,27 & 4,75 & 4,54 & 3,23 & 2,60 & 7,84 & 7,24 & 6,03 & 5,07 \\
\hline \multicolumn{2}{|l|}{ Flow(12mb) } & 2,45 & 1,94 & 2,65 & 4,82 & 3,87 & 3,26 & 2,29 & 7,45 & 7,28 & 5,42 & 4,55 & 11,89 & 11,15 & 9,74 & 8,20 \\
\hline \multicolumn{2}{|l|}{ Flow(18mb) } & 3,25 & 2,61 & 3,52 & 6,20 & 5,12 & 4,41 & 3,18 & 9,56 & 9,44 & 7,19 & 6,18 & 15,02 & 14,18 & 12,68 & 10,69 \\
\hline \multicolumn{2}{|c|}{ Flow(12mb)/Flow(6mb) } & 1,7 & 1,7 & 1,7 & 1,6 & 1,7 & 1,7 & 1,8 & 1,6 & 1,6 & 1,7 & 1,7 & 1,5 & 1,5 & 1,6 & 1,6 \\
\hline \multicolumn{2}{|c|}{ Flow(18mb)/Flow(6mb) } & 2,2 & 2,3 & 2,2 & 2,0 & 2,2 & 2,4 & 2,5 & 2,0 & 2,1 & 2,2 & 2,4 & 1,9 & 2,0 & 2,1 & 2,1 \\
\hline
\end{tabular}

Table 2: This table shows the flow driven for each needle at different starting-point pressure (6 mbar is the design pressure).

\section{First check of the system and conclusions}

A sample of the new gas impedances was installed in the entire gas rack 63 and has been working since the end of November 2011. The gas pressure in the rack inlet has increased to 7.5 mbar (we expect 6 mbar on the new impedance and more than 1 mbar on the rack screw for line regulation).

No problem appeared for the chambers connected to rack 63. Furthermore, a leak test was made by disconnecting the gas inputs in two chambers: the lost flow was compatible with the value of the installed impedance. Given the positive results that we have achieved, we are installing the new gas impedances in all ATLAS RPCs.

\section{References}

[1] De Asmundis, R ATL-MUON-SLIDE-2009-372

[2] Aielli, G Cavern background measurement with the ATLAS RPC system RPC2012 Frascati 